\title{
The effect of acculturation in the concordance of oral health related quality of life in Latino children and their parents
}

\author{
Yltze P. Cubas, Maria F. Orellana
}

Department of Orofacial Sciences, University of California, San Francisco, USA

Email: Orellanam@dentistry.ucsf.edu

Received 15 April 2013; revised 15 May 2013; accepted 21 May 2013

Copyright (C) 2013 Yltze P. Cubas, Maria F. Orellana. This is an open access article distributed under the Creative Commons Attribution License, which permits unrestricted use, distribution, and reproduction in any medium, provided the original work is properly cited.

\begin{abstract}
The Latino population is the fastest growing and the largest minority group in the United States comprising $16 \%$ of the population younger than 18 years of age. Yet, little is known about the effect of acculturation in oral health related quality of life in Latino children and their parents. Objective: The objective of this study was to assess the effect of acculturation in parent and child's perceptions of the child's oral health status and oral health related quality of life (OHRQoL) as well as the effect in the concordance between children and parents/caregivers. Method: Sixty-three Latino children between the ages of 8 and 15 , and their parents were recruited from the waiting room at the University of California, San Francisco Orthodontic and Pediatric Clinics. Parent and children each separately completed the Child Oral Health Impact Profile questionnaire (COHIP) for children and COHIP for parent/caregiver. Results: Of the sixty-three children, fifteen children $(23.8 \%)$ had a more negative perception of their oral health than their parents whereas twenty-three children (36.5\%) had a more positive perception. In terms of agreement between children and parents, questions about oral health showed the lowest level of agreement (34.9\%) and self-image questions the highest (55.6\%). Conclusion: The study findings indicate that the more acculturated the child, the more negative their perception of their oral health. The level of disagreement between parent and child, underscores the importance of obtaining both the child and parent OHRQoL perceptions.
\end{abstract}

Keywords: Oral Health Related Quality of Life

*This project was supported by the CTST/T-32 DE017249-04.
(OHRQoL); Children; Child Oral Health Impact Profile (COHIP); Acculturation; Concordance; Perception

\section{INTRODUCTION}

In the last two decades, a number of instruments have been developed to measure the functional and psychosocial outcomes of oral disorders, most of them only suited for the adult population [1-3]. Measurements of child health status and health related quality of life (HRQoL) were based on proxy reports by parents or caregivers [4] because of the difficulties of developing and validating such measurements for children.

A number of newly developed instruments have shown that it is possible to obtain valid and reliable information when the appropriate questionnaire technique is used with a population of minors $[3,5,6]$. One of these instruments is the Child Oral Health Impact Profile (COHIP) [5,7].

It has been shown that children's understanding of complex concepts, such as health and well-being is affected by variables such as gender, age and the age-related experience to which they are subjected $[8,9]$. It is reasonable then to speculate that cultural norms will influence children's perceptions of their own oral health. In the case of immigrants, levels of acculturation will play an important role.

Acculturation is the multidimensional and multidirectional process whereby immigrants and their descendents adopt the behaviors, believes, and values of the host culture while adapting those belonging to their culture of origin [10]. Significant changes in morbidity and health behavior are associated with acculturation [11]. For example, in Latinos, acculturation plays a role in hypertension [12], diet [13]; smoking among children, adolescent and adults [14,15]; and use of professional dental cleaning and dental care services [16]. In children and adoles- 
cents, differences across racial/ethnic groups have also been shown for behaviors such as bicycle helmet use, seat belt use and eating a healthy diet [17].

In 2000, 1 in 5 children in the US were immigrants or a child of an immigrant, yet the oral health and oralhealth behavior of this large and rapidly growing population remain understudied $[15,18]$.

Parents and/or caregivers are intimately involved in the oral health and the health care of their children. They are usually the principal decision makers in regards to their child's health and treatment of choice. Treatment of children's oral health problems is as likely to be influenced by parental perceptions of their child's needs as it is by the actual needs of the child.

With the creation of the child-specific instruments, a great deal of attention is given to parent versus child reports. Results from studies evaluating this question are ambiguous and appear to depend on the health domain being examined [19-22]. Parents' responses may reflect their own perception of their children's health and may diverge from their child's. We hypothesize that culture and, in the case of immigrants, acculturation will have an impact on parents and children OHRQoL perceptions [23].

To date, no one has examined the effect of acculturation in OHRQoL in Latino children and their parents/ caregivers or the impact of acculturation in the concordance between them. Thus, in this study, we examined child and parental perceptions of the child OHRQoL and their concordance in light of their acculturation levels. Understanding the effects of acculturation on Latino oral health may improve our understanding of oral health disparities in the US.

\section{METHODS}

\subsection{Sampling and Procedures}

For this study, we used a random convenience sample of 63 dyads (parent/children). Parents and children were recruited from the dental clinics at the University of California San Francisco (UCSF). Most of the children were seeking dental treatment at the UCSF Orthodontic and Pediatric Dentistry clinics. Some children were waiting at the clinics with their parents for their siblings to receive treatment. We didn't include these children as orthodontic or pediatric dentistry patients; rather, we created a separate category (other) for them. Inclusion/ex- clusion criteria are shown in Table 1. A trained, bilingual research assistant approached patients who were previously identified as Latino by the reception desk in each clinic. After describing the purpose of this study, interested participants were invited to complete the study questionnaire with the option of completing it either in English or Spanish upon signing of consent/assent forms. For participating in this study a monetary incentive was given. During completion of the questionnaires, participants were seated in a designated quiet area separated from the other member of their dyad and a bilingual research assistant was available to answer any questions. This protocol was approved by UCSF Committee of Human Research \# H60232-33032-01.

\subsection{Instrument}

For this study, we used the COHIP questionnaire [7]. Briefly, it consists of 34 items comprising five domains that assess oral health, functional well-being, social-emotional well-being, school environment, and self-image. COHIP was designed to measure self-reported OHRQoL of children between ages 8 and 15 years old using both positively and negatively worded items. The statements were formatted to elicit self-reports from the child and the parent. Instruction for the questionnaires were: "Please read carefully each statement and choose the answer that best describe you in the past 3 months regarding your teeth, mouth and face" Responses were recorded as "never" $=0$, "almost never" $=1$, "sometimes" $=2$, "fairly often" $=3$, and "almost all the time" $=4$. Questions 31 to 39 were worded in the following format: "strongly agree" $=0$, "somewhat agree" $=2$, "don't agree or disagree" $=3$, "somewhat agree" $=4$ and "strongly agree" $=5$. Scores of the COHIP can range from 0 - 136 .

Demographic questions such as age, gender, number of siblings, parent's level of education, country of birth, geographic area of residence, years living in the Bay Area, household income and dental insurance or method of payment were placed at the beginning of the COHIP questionnaires. A short acculturation scale for Hispanics and a short acculturation scale for the Hispanic youth were also added to the COHIP questionnaire. The acculturation scales for Hispanics [24] consisted of five questions while the acculturation for the Hispanic youth consisted of 12 questions [25] (See appendix 1 for complete questionnaires).

Table 1. Inclusion and exclusion criteria for eligibility.

\begin{tabular}{cc}
\hline Inclusion & Exclusion \\
\hline Children's age 8 to 15 years. & Parent can't provide written consent. \\
At least one parent is identify as Latino & $\begin{array}{c}\text { Previous Orthodontic treatment for child. } \\
\text { Previous orthodontic consult for child. }\end{array}$ \\
Parent and child are able to understand English or Spanish. & $\begin{array}{c}\text { Child has syndromic condition affecting the mouth and face. } \\
\text { Child has mental developmental disability. }\end{array}$ \\
\hline
\end{tabular}




\subsection{Statistical Analysis}

Subscales scores of the COHIP were calculated by summing the responses of the items specific to the subscale. The overall OHRQoL score was computed by summing all the subscales added at the end of the COHIP questionnaires [7]. The overall acculturation scores were calculated by summing all the responses of the acculturation questionnaire $[24,25]$.

Discrepancy scores for each subscale and the overall COHIP score were computed for each dyad (parent/ child). The child score was subtracted from the caregiver score. This yielded a difference score that could be either negative (higher child report of QoL) or positive (higher caregiver report of QoL). Higher COHIP scores reflect more positive OHRQoL; while lower scores reflect lower OHRQoL.

The acculturation score was computed for each dyad (parent/child). Subsequently, the child score was subtracted from the caregiver score.

The differences for each subscale and the overall score were categorized separately using the standard deviation of the dyad's (child and caregiver) difference score for the specific subscale or overall score. Dyads with raw discrepancy scores ranging from one-half standard deviation above zero to one half standard deviations below zero were classified as having similar reports (similar). Those with raw difference scores greater than one half standard deviation below zero were classified as children reporting greater QoL or more acculturated than the caregiver (child more positive). Those with a raw difference score greater than one-half a standard deviation above zero were classified as caregivers reporting greater QoL or less acculturated than the child (child more negative).

\subsection{Krustal-Wallis Testing}

A non-parametric method for testing equality of population median among groups was performed to compare the concordance between categories and clinical groups for the overall COHIP score, as well as the individual subscales and the acculturation scores. It was also used to compare concordance categories and child gender and child age (dichotomized in 12 years or younger and older than 12 years). The sum of the ranks was calculated for each group, then the test statistic, $\mathrm{H}$, was calculated. Subsequently, a pair-wise multiple comparisons was constructed to locate the source of significance.

\section{RESULTS}

Sixty three children and their parents completed the COHIP and acculturation questionnaires. Table 2 shows the demographic characteristics of the sample. Seventy
Table 2. Demographic characteristics of the dyads.

\begin{tabular}{cccc}
\hline \multicolumn{2}{c}{ Variable } & Children & Parents \\
\hline \multicolumn{2}{c}{ Age ( Mean \pm SD) } & $10.8 \pm 2.2$ & $38.8 \pm 7.2$ \\
\hline \multicolumn{2}{c}{ Age (Median (min-max)) } & $11.0(7.0-16.0)$ & $37.0(26.0-54.0)$ \\
\hline \multirow{2}{*}{ Gender } & Male & $31(49.2 \%)$ & $12(19 \%)$ \\
& Female & $32(50.8 \%)$ & $51(81 \%)$ \\
\hline \multirow{2}{*}{ Country of } & US & $50(79.4 \%)$ & $12(19 \%)$ \\
Birth & Mexico & $7(11.1 \%)$ & $31(49.2 \%)$ \\
& El Salvador & $3(4.8 \%)$ & $9(14.3 \%)$ \\
& Other & $3(4.8 \%)$ & $11(17.5 \%)$ \\
\multirow{4}{*}{ Payer } & Medical & $34(54 \%)$ \\
& Priv. Insurance & $7(11.1 \%)$ \\
& Self & $9(14.3 \%)$ \\
Site & Healthy family & $8(12.7 \%)$ \\
& Other & $5(7.9 \%)$ \\
\hline & Pediatric & $28(44.4 \%)$ \\
& Orthodontics & $21(33.3 \%)$ \\
\hline
\end{tabular}

nine percent of the children were born in the United States; while only $19 \%$ of parents were born in Unites States. The sampled children ranged in age from 7 to 16 years old. Parents' age ranged from 26 to 54 years, average of $37(\mathrm{SD}=7.2)$. Most of these children had governmental supported dental insurance, Medi-Cal (54\%). Parents level of education averaged $10.9(\mathrm{SD}=3.6$; Median $=12$ ).

Only $3(4.8 \%)$ children were less acculturated than their parents, $17(27 \%)$ had similar levels of acculturation and $43(68 \%)$ children were more acculturated than their parents. The difference of acculturation between children and parents had an average of $1.2(\mathrm{SD}=1.2$; Median $=1.2$, $\min -\max =-1.5$ to 6.6 ) which indicated that the more acculturated the child the more negative their perception of their own teeth in general compared with their parents.

Overall COHIP results from the concordance between the COHIP total scores versus concordance in acculturation are depicted in Table 3. Thirty six percent of children were more likely to rate a more negative OHRQoL compared with their dyads.

\subsection{COHIP by Domain}

\subsubsection{Oral Health}

The proportion of disagreement between children and their parents showed that the child was more likely to rate oral health more negative $(41 \%)$ than their parent $(24 \%)$ P-value $=0.56$.

\subsubsection{Functional Well-Being}

The proportion of disagreement between children and their parents showed that the child was less likely to rate 
functional well-being subscale more negative $(19 \%)$ than their parent $(30 \%)$, P-value $=0.54$.

\subsubsection{Social Emotional Well-Being}

The proportion of disagreement between the children and their parents showed that the child was more likely to rate social well-being more negative $(33 \%)$ than their parent $(27 \%)$, P-value $=0.36$.

\subsubsection{School Environment}

The proportion of disagreement between the children and their parent showed that the child was more likely to rate the school environment more negative $(32 \%)$ than their parent $(19 \%)$, P-value $=0.95$.

\subsubsection{Self-Image}

The proportion of disagreement between the children and their parent showed that the child was more likely to rate self-esteem more negative (28\%) than their parent (16\%), $\mathrm{P}$-value $=0.33$.

Other predictors of interest such as the difference between parents and children social emotional scores were also analyzed (Table 4). A univariate linear regression analysis showed that the number of years the parents lived in the United States (one proxy for acculturation) was statistical significantly associated to social emotional scores $(\mathrm{P}$-value $=0.040)$. Differences between acculturation level of the parent, $\mathrm{P}$-value $=0.063$, and acculturation level of the children, P-value $=0.094$, were found, although not statistical significant.

Additionally, the difference between parent's and child's self-image score was 6.8 points less for children born in other country other than US or Mexico; P-value $=0.034$ (Table 5). Differences between acculturation level of the

Table 3. Differences in total COHIP scores and differences in parental and child acculturation levels.

\begin{tabular}{|c|c|c|c|c|c|c|}
\hline \multirow{2}{*}{\multicolumn{2}{|c|}{ Variable }} & \multicolumn{3}{|c|}{$\begin{array}{l}\text { Difference in COHIP Scores: } \\
\text { OHRQoL perceptions }\end{array}$} & \multirow{2}{*}{ Total } & \multirow{2}{*}{ P-value } \\
\hline & & $\begin{array}{l}\text { More Positive } \\
15(24 \%)\end{array}$ & $\begin{array}{l}\text { Similar } \\
25(40 \%)\end{array}$ & $\begin{array}{c}\text { More negative } \\
23(36 \%)\end{array}$ & & \\
\hline \multirow{3}{*}{$\begin{array}{c}\text { Acculturation } \\
\text { (child compared to parent) }\end{array}$} & Less Acculturated & $0(0 \%)$ & $3(100 \%)$ & $0(0 \%)$ & $3(4.8 \%)$ & \multirow{3}{*}{$\begin{array}{c}\mathrm{P}=0.36 \\
\text { Based on Fisher exact test }\end{array}$} \\
\hline & Similar & $5(29.4 \%)$ & $7(41.2 \%)$ & $5(29.4 \%)$ & $17(27 \%)$ & \\
\hline & More Acculturated & $10(23.2 \%)$ & $15(34.9 \%)$ & $18(41.9 \%)$ & $43(68.2 \%)$ & \\
\hline
\end{tabular}

Table 4. Univariate linear regression of differences on the social-emotional score.

\begin{tabular}{|c|c|c|c|c|c|c|c|}
\hline \multirow{2}{*}{\multicolumn{2}{|c|}{ Variable }} & \multicolumn{3}{|c|}{ Children } & \multicolumn{3}{|c|}{ Parent } \\
\hline & & \multirow{2}{*}{$\begin{array}{c}\text { Estimate } \\
0.000\end{array}$} & \multirow[t]{2}{*}{$95 \% \mathrm{CI}$} & \multirow[t]{2}{*}{ P-value } & \multirow{2}{*}{$\begin{array}{c}\text { Estimate } \\
0.000\end{array}$} & \multirow[t]{2}{*}{$95 \%$ CI } & \multirow[t]{2}{*}{ P-value } \\
\hline C ond & Male & & & & & & \\
\hline Gender & Female & -0.92 & $-4.67-2.8$ & 0.63 & -0.34 & $-5.13-4.5$ & 0.89 \\
\hline \multirow{3}{*}{$\begin{array}{l}\text { Country } \\
\text { of Birth }\end{array}$} & US & 0.000 & & & 0.000 & & \\
\hline & Mexico & -0.47 & $-6.50-5.6$ & 0.88 & 0.37 & $-5.7-6.5$ & 0.90 \\
\hline & Other & 0.43 & $-6.02-6.9$ & 0.89 & 2.5 & $-1.63-6.6$ & 0.23 \\
\hline \multicolumn{2}{|c|}{ Age } & 0.97 & $0.16-1.78$ & 0.019 & 0.03 & $-0.23-0.29$ & 0.83 \\
\hline \multicolumn{2}{|c|}{ Acculturation } & 1.86 & $-0.33-4.1$ & 0.094 & 1.43 & $-0.08-2.9$ & 0.063 \\
\hline & & \multicolumn{2}{|c|}{ Estimate } & \multicolumn{2}{|c|}{$95 \%$ CI } & \multicolumn{2}{|c|}{ P-value } \\
\hline \multirow{5}{*}{ Site } & Pediatric & \multicolumn{2}{|c|}{0.000} & & & & \\
\hline & Orthodontic & \multicolumn{2}{|c|}{5.5} & \multicolumn{2}{|c|}{$1.51-9.5$} & \multicolumn{2}{|c|}{$0.007^{*}$} \\
\hline & Other & \multicolumn{2}{|c|}{-1.36} & \multicolumn{2}{|c|}{$-5.88-3.2$} & \multicolumn{2}{|c|}{0.55} \\
\hline & Self & \multicolumn{2}{|c|}{0.000} & & & & \\
\hline & Medical & \multicolumn{2}{|c|}{-1.98} & \multicolumn{2}{|c|}{$-7.12-3.2$} & \multicolumn{2}{|c|}{0.44} \\
\hline \multirow[t]{3}{*}{ Payer } & Healthy Family & \multicolumn{2}{|c|}{-9.96} & \multicolumn{2}{|c|}{$-16.6--3.3$} & \multicolumn{2}{|c|}{$0.004^{*}$} \\
\hline & Other & \multicolumn{2}{|c|}{0.67} & \multicolumn{2}{|c|}{$-6.97-8.3$} & \multicolumn{2}{|c|}{0.86} \\
\hline & Private & \multicolumn{2}{|c|}{-1.48} & -8.38 & -5.4 & & \\
\hline Parer & tion & & .16 & -0.36 & -0.69 & & \\
\hline Parent's ye & in the US & & .15 & 0.007 & -0.30 & & \\
\hline
\end{tabular}


Table 5. Univariate linear regression of differences on the self image score.

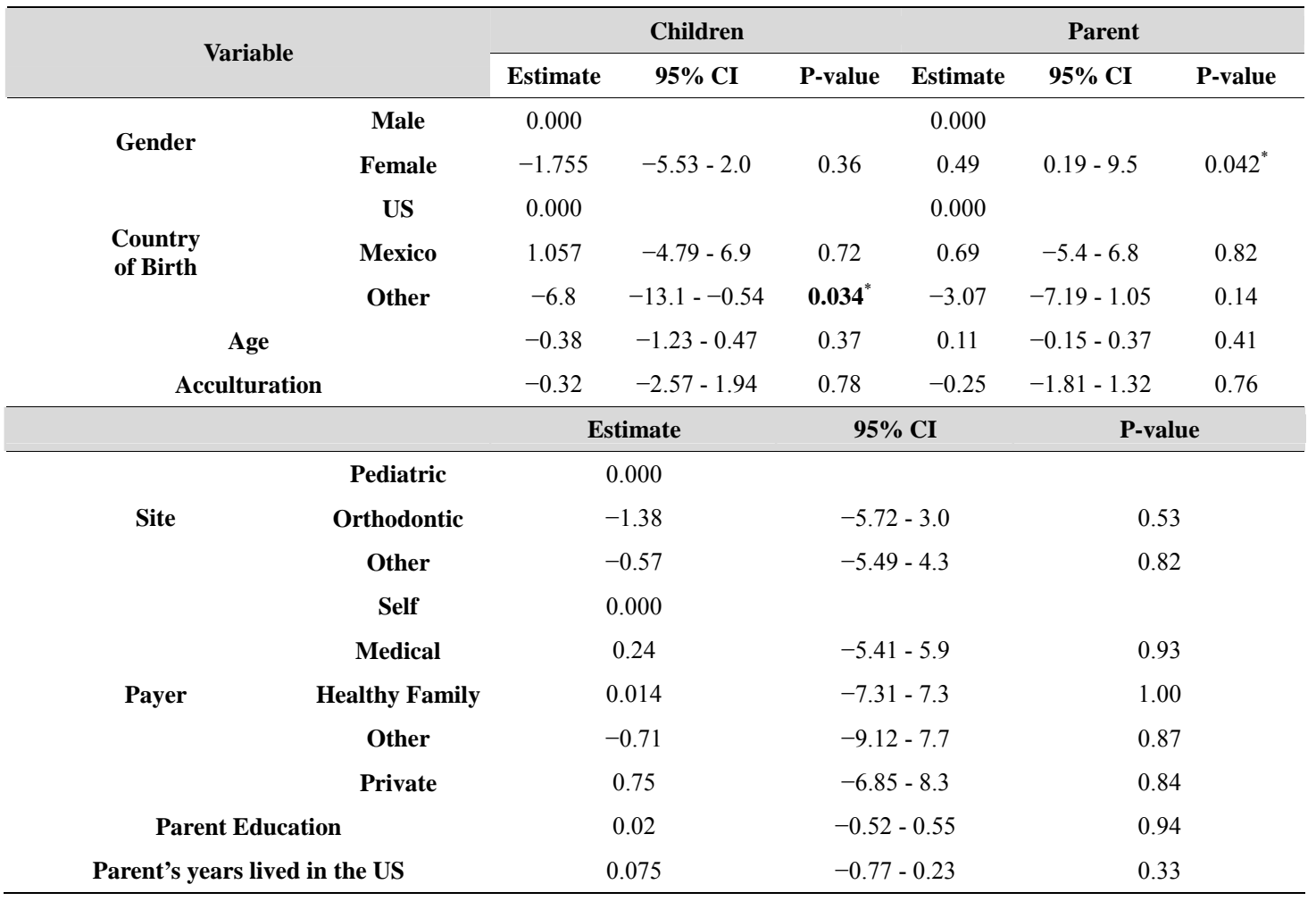

children and acculturation level of the parent were also found, however not statistically significant.

\section{DISCUSSION}

This study was conducted to explore the effect of acculturation in parent/caregiver and children's perception of the child's oral health related quality of life as well as it effects in concordance between Latino children and their parents. Our results showed that there are more children/ adolescents with negative perception than a positive perception compared with similar questions to their dyads, which indicated a poorer perception as it relates to oral health, social emotional, school and self-image, although not statistical significant (Table 6).

This data showed a negative children's perception between the differences in scores by domain of the COHIP and parental/child acculturation levels. These findings suggest that lower agreement possibly occurs when the parent and child have access to different information (school, peer relationships). Parent's responses may reflect the truth as they perceive it, which may not be identical to that of their child. We then believe that culture and, in case of immigrants, acculturation will then have an impact on parents and children OHRQoL perceptions.

Findings have documented that children and parents often fail to agree and this may explain the poor outcomes often reported. Yeh May and et (2001) found that in parent-child pairs, $63 \%$ failed to agree on even a single problem for where the child needed help and when problems were grouped into broad categories more than one third failed to agree on a single broad problem area [26]. This evidence corroborates with Canning et al in that agreements between parent and child are poor; independent of child's medical illness, age, gender, or type of psychiatric disorder [4]. Furthermore, Theunissen and et in 1998 concluded that children reported a significant lower HRQoL than their parents on the physical complaints, motor functioning, autonomy, cognitive functioning and positive emotion scales $(p<0.05)$ [19]. Similarly, Vogels et al. in 1998 found scarcely any agreement on autonomy and social and global emotional functioning: the t-test resulted in only one significant outcome: parents rated their children's mood slightly more positive than their children themselves did [21]. This is an indication that a mismatch of concordance between parents and child is poor regardless of cultural level which concurred with our findings that parent and children see problems with different perceptions and with different interpretations overall.

Although not statistical significant, our data showed that the more acculturated the child the more negative their perception of their own oral health compared with the perception of their parents. Riley in 2008 found lower levels of acculturation, particularly in less frequent use of English, were associated with greater oral pain 
Table 6. Differences in scores by domain and differences in parental and child acculturation levels.

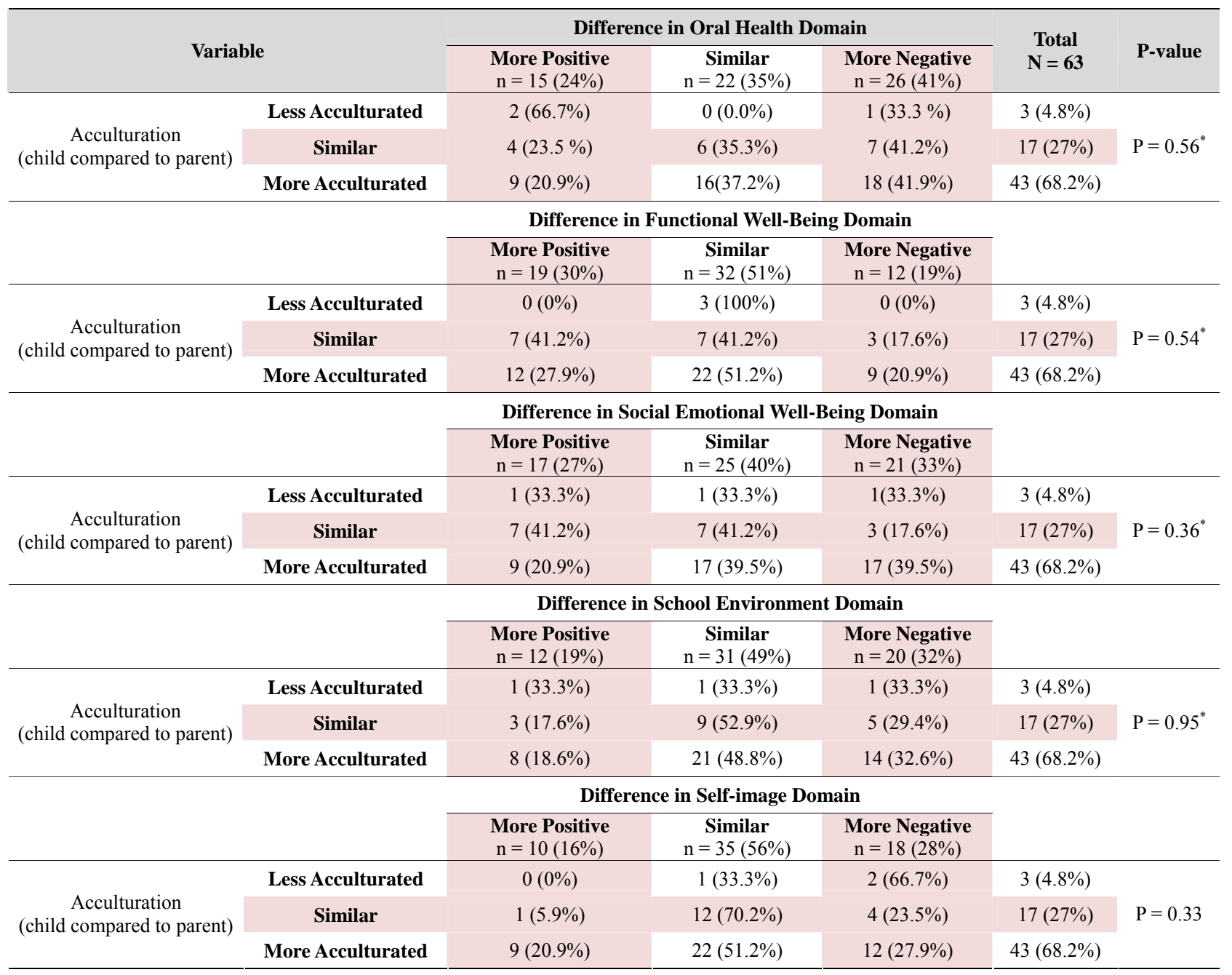

*Based on Fisher exact test.

and depression [27]. Fung, J. \& Lau, A. suggested that parent-child discrepancies in problem identification may be heightened among ethnic minority families, where expectations about appropriate youth and parent behavior may diverge as a function of differential orientations toward ethnic and dominant cultural values. In addition, she suggested that parental acculturative stress and parent-child acculturation dissonance predicted more disagreement regarding internalizing problems among Chinese immigrant families [28]. The distinction between existing problems and emotional reactions towards problems seems to be relevant, in as much as most parents signaling a problem do not report associated negative emotional feelings in their child.

Our results support the hypothesis that greater child acculturation to US culture might predict more disagreement only when parents are less acculturated to the US culture and that this might impact the perception of oral health. Studies done by the office of minority health stated that acculturation influences perceived oral health amongst other in the Hispanic population [29]. Ethnicity seems to also play a role in perception of oral health; for example, racial minorities report lower OHRQoL [30]. In regards to the instrument used in this study, Blacks and Latinos have lower scores for the overall COHIP scores than Whites [5]. Additionally, Dubard in 2008 reported that Spanish speaking individuals were substantially more likely to report poor or fair health status (39\%) than were English speaking hispanics (17\%), a difference that remained significant after adjustment for age, gender and education [31].

We are providing evidence that several factors may impact the difference between children and parent COHIP total scores and subscales scores. We believe this observation warrants further studies with greater sample size as proposed herein. Parent's opinion is no doubt of great importance; parents are generally quite able to estimate their child's well-being. However, parents may 
additionally easily over or underestimate the importance their children's attributes to certain aspects of their wellbeing at a specific point in time; thus, peer related issues may be far more important to an adolescent than parents have ever thought. Moreover, parent's expectations and previous experiences with the child may well influence their views.

These findings underscore the importance of not only using information given by the parents themselves, but also communicating with the child and their parents as a part of clinical practice. Since children's reports have demonstrated to be valid and reliable; children's questionnaires should be used as an outcome measure especially among immigrant families.

\section{Limitations of the Study}

First, the sample size which was not large enough to determine if the predictors of OHRQoL varied according to the concordance between parents affecting the children included in this study or the effect of acculturation per se. Therefore, it will be useful to replicate this study in higher sample size.

Second, the study design which was a cross sectional design, though common in acculturation research (including all known risk studies) means potential confounders that can be controled only statistically, limiting causal inferences.

Third, the parents and children who participated in this study had already achieved access to health care, simply by coming to the clinic. Even though we questioned the subjects about acculturation and perceptions of oral health; it is possible that the study's population had a different perception than the general population.

Fourth, the majority of parents of this study were mothers. Although this high percentage can be ecologically valid in reflecting which parents are usually more likely to seek and be involved in the child's health care, it is possible that the mothers differ from fathers and other informants in their level of agreement with the child about major problems.

Fifth, the study method might have bias the results due to the tools used in this study. Some participants are prompted to disclose more information in a face to face interview compared to a written questionnaire. Furthermore, it is unclear how many participants did not have adequate proficiency in English or Spanish; even though, they had the choice of choosing a preference language questionnaire.

Last, reporting bias would occur if a parent thinks that the unmet need is a socially undesirable concept. There is some evidence that social desirability is a particularly important factor influencing how Latinos answer behavioral surveys.

\section{CONCLUSION}

- Acculturation plays a role in the perception of oral health.

- There is a correlation between the level of acculturation and discordance in the report child/caregiver OHRQoL among Latinos.

- Our findings underscore the importance of the caregiver's reports when making diagnostics or treatment decisions concerning Latino children.

- Future research is warranted to further explore the effect of acculturation on the concordance between caregiver and their children.

- Strategies to bridge communication to improve the congruence between parent's and children's perceptions of family and youth problems may present a crucial step in establishing a working alliance within the first visit to family immigrants.

\section{REFERENCES}

[1] Leao, A. and Sheiham, A. (1996) The development of a socio-dental measure of dental impacts on daily living. Community Dental Health, 13, 22-26.

[2] Atchison, K.A. and Dolan, T.A. (1990) Development of the geriatric oral health assessment index. Journal of Dental Education, 54, 680-687.

[3] Jokovic, A., Locker, D., Stephens, M., Kenny, D., Tompson, B. and Guyatt, G. (2002) Validity and reliability of a questionnaire for measuring child oral-health-related quality of life. Journal of Dental Research, 81, 459-463. doi: $10.1177 / 154405910208100705$

[4] Canning, E.H., Hanser, S.B., Shade, K.A. and Boyce, W.T. (1992) Mental disorders in chronically ill children: Parent-child discrepancy and physician identification. $\mathrm{Pe}$ diatrics, 90, 692-696.

[5] Broder, H.L. (2007) Children's oral health-related quality of life. Community Dentistry and Oral Epidemiology, 35 5-7. doi:10.1111/j.1600-0528.2007.00400.x

[6] Broder, H.L., McGrath, C. and Cisneros, G.J. (2007) Questionnaire development: Face validity and item impact testing of the child oral health impact profile. Community Dentistry and Oral Epidemiology, 35, 8-19. doi:10.1111/j.1600-0528.2007.00401.x

[7] Broder, H.L. and Wilson-Genderson, M. (2007) Reliability and convergent and discriminant validity of the child oral health impact profile (COHIP child's version). Community Dentistry and Oral Epidemiology, 35, 20-31. doi:10.1111/j.1600-0528.2007.0002.x

[8] Jokovic, A., Locker, D. and Guyatt, G. (2005) What do children's global ratings of oral health and well-being measure? Community Dentistry and Oral Epidemiology, 33, 205-211. doi:10.1111/j.1600-0528.2005.00210.x

[9] Foster Page, L.A., Thomson, W.M., Jokovic, A. and Locker, D. (2005) Validation of the child perceptions questionnaire (CPQ 11-14). Journal of Dental Research, 84, 649-652. doi: $10.1177 / 154405910508400713$ 
[10] Landrine, H. and Klonoff, E.A. (2004) Culture change and ethnic-minority health behavior: An operant theory of acculturation. Journal of Behavioral Medicine, 27, 527555. doi:10.1007/s10865-004-0002-0

[11] Espino, D.V. and Maldonado, D. (1990) Hypertension and acculturation in elderly mexican americans: Results from 1982-84 hispanic HANES. The Journals of Gerontology, 45, M209- M213. doi:10.1093/geronj/45.6.M209

[12] Gardner, C., Winkleby, M.A. and Viteri, F.E. (1995) Dietary-intake patterns and acculturation levels of hispanic immigrant men-A pilot-study. Hispanic Journal of Behavioral Sciences, 17, 347-361. doi:10.1177/07399863950173005

[13] Otero-Sabogal, R., Sabogal, F., Perez-Stable, E.J. and Hiatt, R.A. (1995) Dietary practices, alcohol consumption, and smoking behavior: Ethnic, sex, and acculturation differences. Journal of the National Cancer Institute, (18), 73-82.

[14] Perez-Stable, E.J., Ramirez, A., Villareal, R., et al. (2001) Cigarette smoking behavior among US latino men and women from different countries of origin. American Journal of Public Health, 91, 1424-1430. doi:10.2105/AJPH.91.9.1424

[15] Legal immigration to California in 2002 Linda Gage, California Department of Finance, Demographic Research Unit.

http://search.ca.gov/search?q=cache:WkelT3cBdq4J:ww w.dof.ca.gov/HTML/DEMOGRAP/ReportsPapers/Immig rationMigration/Legal-02/documents/2002 INS Report. pdf\&proxystylesheet $=$ xfix\&output $=$ xml no $\mathrm{dtd} \&$ client $=$ xfix.

[16] Watson, M.R. and Brown, L.J. (1995) The oral health of US Hispanics: Evaluating their needs and their use of dental services. The Journal of the American Dental Association, 126, 789-795.

[17] Grunbaum, J.A., Kann, L., Kinchen, S., Ross, J., Hawkins, J., Lowry, R., et al. (2003) Youth risk behavior surveillance-United States, (Abridged). Journal of School Health, 2004 OCT; 74, 307-324. doi:10.1111/j.1746-1561.2004.tb06620.x

[18] Flores, G., Fuentes-Afflick, E., Barbot, O., et al. (2002) The health of latino children: Urgent priorities, unanswered questions, and a research agenda. JAMA, 288, 82-90. doi:10.1001/jama.288.1.82

[19] Theunissen, N.C., Vogels, T.G., Koopman, H.M., et al. (1998) The proxy problem: Child report versus parent report in health-related quality of life research. Quality of Life Research, 7, 387-397. doi:10.1023/A:1008801802877

[20] Verrips, G.H., Vogels, A.G., den Ouden, A.L., Paneth, N. and Verloove-Vanhorick, S.P. (2000) Measuring healthrelated quality of life in adolescents: Agreement between raters and between methods of administration. Child: Care, Health and Development, 26, 457-469. doi:10.1046/j.1365-2214.2000.00181.x
[21] Vogels, T., Verrips, G.H., Verloove-Vanhorick, S.P., et al. (1998) Measuring health-related quality of life in children: The development of the TACQOL parent form. Quality of Life Research, 7, 457-465. doi:10.1023/A:1008848218806

[22] Sawyer, M., Antoniou, G., Toogood, I. and Rice, M. (1999) A comparison of parent and adolescent reports describing the health-related quality of life of adolescents treated for cancer. International Journal of Cancer, 12, $39-45$.

doi:10.1002/(SICI)1097-0215(1999)83:12+<39::AID-IJC 8>3.0.CO;2-7

[23] (2008) Number of immigrants Hits Record 37.5MWashingtonpost.com.

http://www.washingtonpost.com/wp-dyn/content/article/2 007/09/12/AR2007091200071.html

[24] Marin, G., Sabogal, F., Vanoss Marin, B., Otero-Sagobal, R. and Perez-Stable, E. (1987) Development of a Short Acculturation Scale for Hispanics. Hispanic Journal of Behavioral Sciences, 9, 183-205. doi:10.1177/07399863870092005

[25] Barona, A. and Miller, J.A. (1994) Short acculturation scale for Hispanic youth (SASH-Y): A preliminary report. Hispanic Journal of Behavioral Sciences, 16, 155-162. doi:10.1177/07399863940162005

[26] Yeh, M. and Weisz, J.R. (2001) Why are we here at the clinic? Parent-child (dis)agreement on referral problems at outpatient treatment entry. Journal of Consulting and Clinical Psychology, 69, 1018-1025. doi:10.1037/0022-006X.69.6.1018

[27] Riley 3rd, J.L., et al. (2008) Acculturation and orofacial pain among Hispanic adults. Journal of Pain, 9, 750-758. doi:10.1016/i.jpain.2008.03.007

[28] Fung, J.J. and Laus, A.S. (2010) Factors associated with parent-child (dis)agreement on child behaviour and parenting problems in Chinese immigrant families. Journal of Clinical Child \& Adolescent Psychology, 39, 314-327. doi:10.1080/153744111003691693

[29] Payne, K.W. and Ugarte, C. A. (1989) The Office of Minority Health Resource Center: Impacting on health related disparities among minority population. Health Education, 20, 6-8.

[30] Beltran-Aguilar, E.D., Barker, L.K., Canto, M.T., Dye, B.A., Gooch, B.F., Griffin, S.O., et al. (2005) Surveillance for dental caries, dental sealants, tooth retention, edentulism, and enamel fluorosis-United States, 19881994 and 1999-2002. MMWR Surveillance Summaries, 54, 1-43.

[31] Dubard Annette, C. and Gizlice, Z. (2008) Language spoken and differences in health status, access to care, and receipt of preventive services among US Hispanics. American Journal of Public Health, 98, 2021-2028. doi:10.2105/AJPH.2007.119008 


\section{APPENDIX I.}

\section{Child's Questionnaire}

In the past 3 months, how often have you

1) Had pain in your teeth/toothache?

2) Been breathing through your mouth or snoring?

3) Had discolor teeth or spot in your teeth?

4) Had crooked teeth or spaces between your teeth?

4) Had sores or sores spots in or around your mouth?

6) Had bad breath?

7) Had bleeding gums?

8) Had food sticking in or between your teeth?

9) Had dry mouth?

10) Had dry mouth or lips?

11) Had trouble biting off or chewing foods such as apple, carrot or firm?

12) Been unhappy or sad because of your teeth, mouth, or face?

13) Missed school for any reason because of your teeth, mouth or face?

14) Been confident because of your teeth, mouth or face?

15) Had difficulty eating foods you would like to eat because of your teeth, mouth or face?

16) Felt worried or anxious because of your teeth, mouth or face?

17) Felt shy or withdrawn because of your teeth, mouth or face?

18) Had difficulty paying attention in school because of your teeth, mouth or face?

19) Avoided smiling or laughing with other children because of your teeth, mouth or face?

20) Had trouble sleeping because of your teeth, mouth or face?

21) Not wanted to speak/read out loud in class because of your teeth, mouth or face?

22) Been teased, bullied or called names by other children because of your teeth, mouth or face?

23) Had difficulty saying certain words because of your teeth, mouth or face?

24) Felt that you look different because of your teeth, mouth or face?

25) Had people have difficulty understanding what you were saying because of your teeth, mouth or face?

26) Felt that you were attractive (good looking) because of your teeth, mouth or face?

27) Had difficulty keeping your teeth clean because of your teeth, mouth or face?

28) Been worried about what other people think about your teeth, mouth or face?

29) Been upset or uncomfortable with being asked questions about your teeth, mouth or face?

30) Not wanted to go to school because of your teeth, mouth or face?

31) I have good teeth.

32) When I am older, I believe (think) that I will have good teeth.

33) When I am older, I believe I will have good health.

34) I feel good about myself.

35) I will feel better about myself when treatment that I need for my teeth, mouth and face.

36) I am nervous (anxious) about the treatment that I need for my teeth, mouth or face.

37) I need orthodontic treatment (braces).

38) I am nervous (anxious) about having braces.

39) Overall I feel my health is: (Poor, Fair, Average, Good, Excellent).

40) What language(s) do you read and speak?

41) What language(s) do your parents speak to you in?

42) What language(s) do you usually speak at home?

43) In which language do you usually think?

44) In which language(s) do you usually speak to your friends in?

45) In what languages(s) are the TV programs you usually watch?

46) In what language(s) are the radio programs you usually listen to?

47) In what language(s) are the movies, TV, and radio programs you want to watch or listen to?

48) In what language(s) do you parents speak to their parents?

49) Your close friends are?

50) You like going to parties at which people are? 
51) The people you visit or who visit you are...

\section{Parent's Questionnaire}

During the last 3 months. How often has your child

1) Had pain in his/her teeth/toothache?

2) Been breathing through his/her mouth or snoring?

3) Had discolor teeth or spot in his/ her teeth?

4) Had crooked teeth or spaces between his/ her teeth?

5) Had sores or sores spots in or around his/her mouth?

6) Had bad breath?

7) Had bleeding gums?

8) Had food sticking in or between his/her teeth?

9) Had pain or sensitivity in his/her teeth with hot or cold things?

10) Had dry mouth or lips?

11) Had trouble biting off or chewing foods such as apple, carrot or firm?

12) Been unhappy or sad because of his/her teeth, mouth, or face?

13) Missed school for any reason because of his/her teeth, mouth or face?

14) Been confident because of his/her teeth, mouth or face?

15) Had difficulty eating foods he/she would like to eat because of your teeth, mouth or face?

16) Felt worried or anxious because of his/her teeth, mouth or face?

17) Felt shy or withdrawn because of his/her teeth, mouth or face?

18) Had difficulty paying attention in school because of his/her teeth, mouth or face?

19) Avoided smiling or laughing with other children because of his/her teeth, mouth or face?

20) Had trouble sleeping because of his/her teeth, mouth or face?

21) Not wanted to speak/read out loud in class because of his/her teeth, mouth or face?

22) Been teased, bullied or called names by other children because of his/her teeth, mouth or face?

23) Had difficulty saying certain words because of his/her teeth, mouth or face?

24) Felt that you look different because of his/her teeth, mouth or face?

25) Had people have difficulty understanding what he/she were saying?

26) Felt that he/she was attractive (good looking) because of your teeth, mouth or face?

27) Had difficulty keeping his/her teeth clean because of your teeth, mouth or face?

28) Been worried about what other people think about his/her teeth, mouth or face?

29) Been upset or uncomfortable with being asked questions about his/her teeth, mouth or face?

30) Not wanted to go to school because of his/her teeth, mouth or face?

31) $\mathrm{He} / \mathrm{she}$ has good teeth?

32) When he/she is older, he/she believes (thinks) that he/she will have good teeth.

33) When he/she is older, he/she believes (thinks) that he/she will have good health.

34) $\mathrm{He} / \mathrm{she}$ feels good about herself.

35) He/she will feel better about him/herself when the treatment for his/her teeth, mouth and face is completed.

36) $\mathrm{He} / \mathrm{she}$ is nervous (anxious) about the treatment that he/she needs for his/her teeth, mouth or face.

37) Overall I feel his/her health is...

38) Overall I feel his/her oral health is...

39) In general, what language do you read and speak?

40) What was the language you used as a child?

41) What language do you usually speak in your home?

42) In what language do you usually think?

43) What language do you usually speak with your friends? 